\title{
CT-guided percutaneous laser ablation of metastatic lung cancer: three cases report and literature review
}

\author{
Qiyu Zhao', Guo Tian², Fen Chen ${ }^{3}$, Liyun Zhong ${ }^{1}$ and Tian'an Jiang ${ }^{1}$ \\ ${ }^{1}$ Department of Ultrasonography, First Affiliated Hospital, Zhejiang University School of Medicine, Hangzhou China \\ 2 State Key Laboratory for Diagnosis and Treatment of Infectious Diseases, Collaborative Innovation Center for Diagnosis \\ and Treatment of Infectious Diseases, The First Affiliated Hospital, Zhejiang University School of Medicine, Hangzhou, China. \\ ${ }^{3}$ Department of Hepatobiliary Pancreatic Surgery, First Affiliated Hospital, Zhejiang University School of Medicine, Hangzhou, \\ China
}

Correspondence to: Tian'an Jiang, email: tiananjiang@126.com

Keywords: laser ablation; CT-guided; computed tomography; lung; lung cancer

Received: September 02, 2016 Accepted: November 22, 2016

Published: December 10, 2016

\section{ABSTRACT}

Objective: To report the efficacy and safety of CT-guided percutaneous laser ablation (PLA) for metastatic lung tumors.

Methods: Three cases of metastatic lung cancer underwent CT-guided PLA, and we searched for previously published articles on the minimally invasive CT-guided RFA or MWA for lung tumors in recent five years.

Results: With the guidance of CT, all lesions had good prognosis under laser ablation. Case 1 suffering from severe pulmonary dysfunction and diffuse pulmonary bullae, had small pneumothorax. CT scan obtained four months following the ablation showed two lesions had complete responses and one partial response. Case $\mathbf{2}$ had successful complete response with absent lung mass, and also had a good postoperative condition without any discomfort in the two-month follow-up. Case 3 showed partial response and improved greatly after five months. 962 cases (mean age of 45.7 years, $62.2 \%$ male) of 1297 lung tumors with detailed information were identified from 27 articles. Of these cases, the minority manifested complications such as pneumothorax, hemoptysis, hemothorax, pneumonia, pain and fever.

Conclusions: Percutaneous CT-guided PLA could be a safe and promising minimally invasive treatment for patients with primary lung cancer or unresectable pulmonary metastases, especially multineedle PLA in large tumors, which still needs more large-scale prospective studies to convince this method in the future.

\section{INTRODUCTION}

Lung cancer is the most common cause of cancerrelated death in the world. It was reported that 226,160 new patients of lung cancer was diagnosed in 2012, and it was evaluated that 160,340 deaths were related to lung cancer [1]. Smoking is the most dangerous factor for lung cancer. Environmental and occupational exposures to carcinogens also have important impacts on this disease. Meanwhile, lung is the second most common site of metastases for colon cancer after liver and traditionally, surgery is the main treatment option. However, it is more expensive and has higher complication risks such as atrial fibrillation, prolonged air leaks, myocardial infarction, recurrent nerve injury, bleeding, pneumonia, and bronchial stenosis [2, 3]. About $10 \%$ to $15 \%$ patients who had a surgical resection for colorectal cancer may develop lung metastasis [4]. In recent years, emerging studies have displayed that CT-guided RFA and MWA are feasible for patients with primary lung cancer or unresectable pulmonary metastases, in which pneumothorax, hemoptysis, pneumonia, pleural effusion may occur [5-22]. It was reported that the 3-year overall survival rate following RFA for pulmonary metastases was nearly $50 \%$ against that $(60 \%)$ of patients treated with pulmonary metastasectomy [4]. However, RFA have a higher impedance in the lung than in the liver, which means poor energy was spread due to high impedance and charing, thus it is difficult to identify whether the lesions are completely ablated or not. 
Additionally, percutaneous laser ablation (PLA) is minimally invasive by introducing the position of laser catheters into the lesions, followed by thermal ablation of targeted tumors through laser energy. PLA has more advantages than other ablative methods including the lower risk of bleeding, infection or injuries of adjacent organs [23], which enable PLA to be valuable for the treatment of small tumors adjacent to vital but subtle regions.

Recently, PLA has been increasingly applied in the treatment of benign and malignant diseases in the liver and thyroid through the guidance of ultrasonography (US) as well as Magnetic Resonance Imaging (MRI) [24-28]. In particular, CT-guided PLA showed a safe, minimally invasive and promising therapy in some lesions such as adrenal metastases and osteoid osteomas [23, 29]. In previous study, percutaneous CT-guided PLA of lung tumors presented complete ablation of lung metastases and lung cancers with the average energy of $46 \mathrm{~kJ}$ (18-102 $\mathrm{kJ})$ through 9F trocar, of which pneumothorax, endothelial hemorrhage and pain were recorded [30, 31]. Here we reported three cases with lung tumors treated by PLA with fine needle and lower energy. We successfully applied CT-guided laser ablation into the lesions in the lung, and postoperative follow-up showed good results.

\section{CASE PRESENTATION}

We retrospectively collected three patients who underwent CT-guided PLA for liver metastatic lung cancer from January 2016 to March 2016 at The First Affiliated Hospital, College of Medicine, Zhejiang University. We targeted these three patients who were evaluated according to the CT imaging. Surgery and chemotherapy were carried out in two patients due to sigmoid colon metastases. The study was approved by the ethics committee of The First Affiliated Hospital of Zhejiang University. These patients had written informed consent for the use of their clinical data and tissues sample for study. Case 1 was a 62-yearold man, who underwent surgical resection of the caudate lobe in March 2013, and two years later, he had TACE in our hospital. Preoperative CT image revealed three lung lesions of $15.4 * 10.7 \mathrm{~mm}, 14.5 * 7.7 \mathrm{~mm}$ and $5 * 5 \mathrm{~mm}$, respectively. This patient could not accepted surgical resection, RFA, MWA or particle implantation treatment because of severe pulmonary dysfunction and diffuse pulmonary bullae. Therefore, CT-guided PLA procedure was conducted. Case 2 was a 64 -year-old man with only one nidus. He had a history of the sigmoid colon tumor resection in 2011, and gamma knife surgery of liver cancer in 2013. Computed tomography detected a $11.6 * 7.5 \mathrm{~mm}$ nodule in the right lung. He also underwent a CT-guided PLA procedure within two laser fibers, each of $1800 \mathrm{~J}$. Case 3 was a 63-year-old woman with sigmoid colon metastatic lung cancer, who had the sigmoid colon tumor and liver metastases resection in 2014. CT revealed a $13 * 9$ $\mathrm{mm}$ lesion of the right-middle lobe.

\section{CT-GUIDED PERCUTANEOUS LASER ABLATION}

The procedures were performed under local anesthesia, in which lidocaine $2 \%$ was administered. Computed tomography (CT) was implemented to

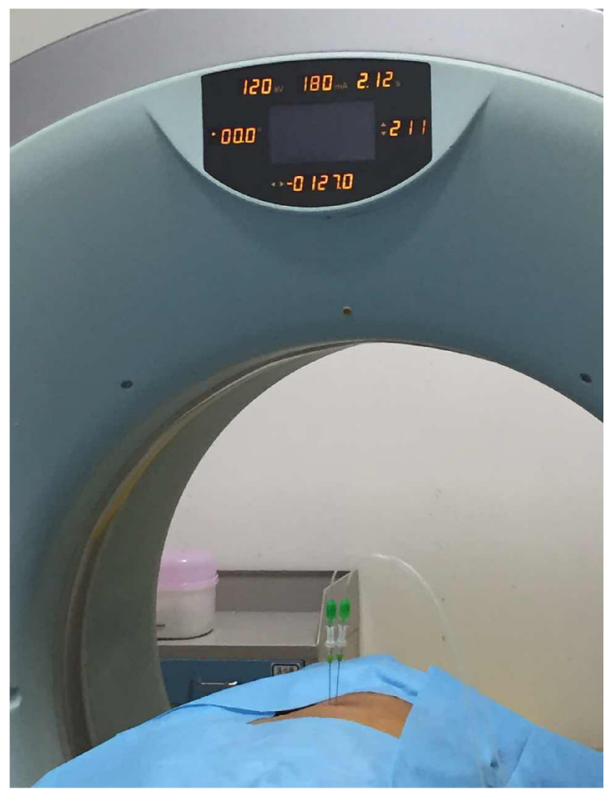

A

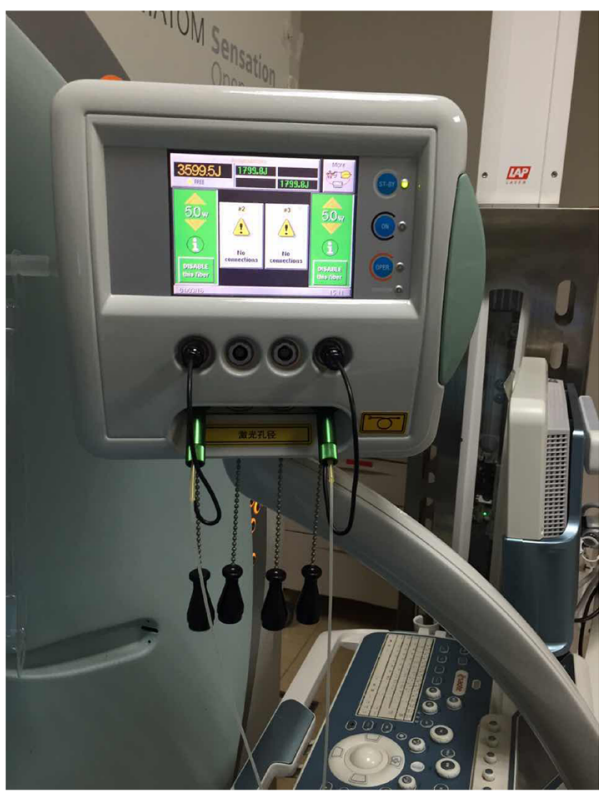

B

Figure 1: Mechanisms of CT-guided percutaneous laser ablation. 
Table 1: Case and tumor characteristics

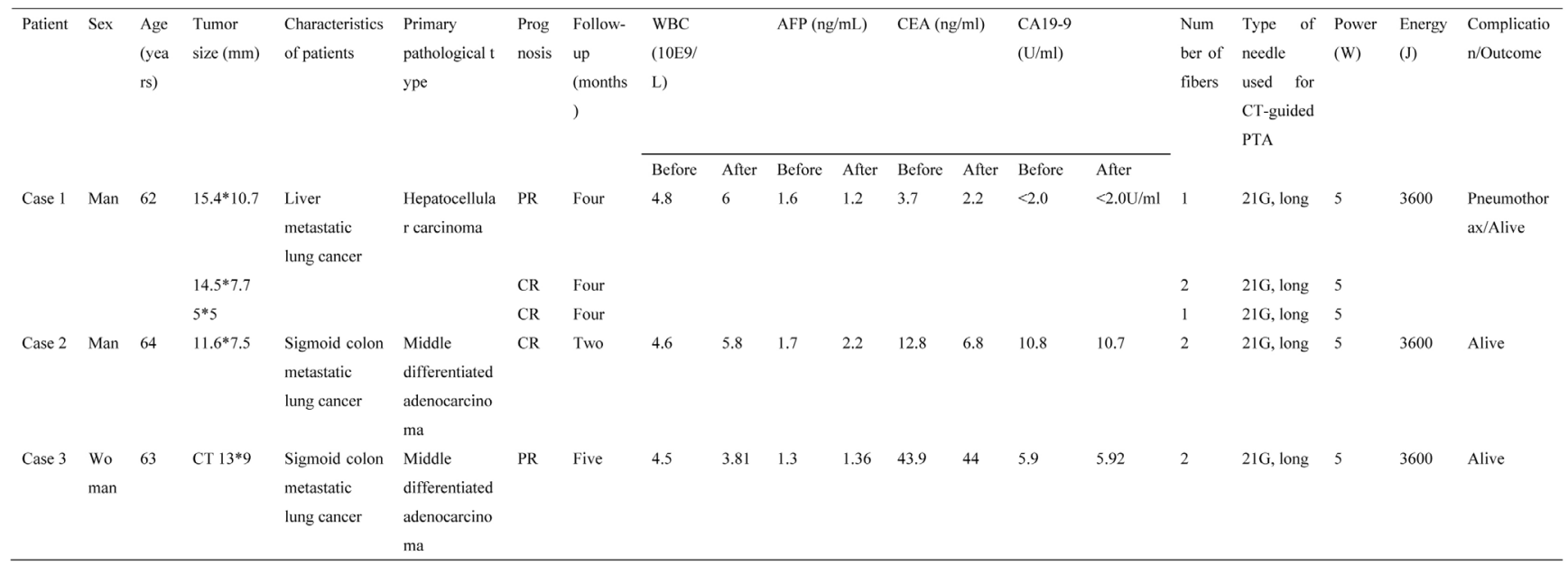

WBC: white blood count;

AFP: alpha fetal protein;

CA199: carbohydrate antigen 199;

CEA: carcino embryonie antigen;

PLA: percutaneous laser ablation;

PR: partial response;

CR: Complete response.

localize the nidus by 64-slice systems (Brilliance iCT and 64-channel systems). With a 21-gauge Chiba needle (Top, Tokyo, Japan), the interventional radiologist withdrew the needle core and placed the laser fiber into the needle sheath outside of the pin end within $1 \mathrm{~cm}$. If the tumor is larger, we can ablate it simultaneously with at most 4 laser fibers. The nidus was ablated by a Nd:YAG laser fiber with a wavelength of $1064 \mathrm{~nm}$, each of which has an output power of $5 \mathrm{~W}$ for $1800 \mathrm{~J}$ (Echolaser X4, ESAOTE, Italy) (Figure 1A and 1B). Targeted tumour tissue would be in coagulation necrosis when the tissue temperature reached $55^{\circ} \mathrm{C}$. When there was residual area left, an additional laser ablation should be conducted subsequently.

\section{FOLLOW-UP AND OUTCOME MEASURES}

The outcome of the local ablation was evaluated according to the World Health Organization (WHO) response evaluation criteria for solid tumor: 1) Complete response (CR): disappearance of all target niduses without any residual lesion for a month; 2) Partial response (PR): $50 \%$ or more reduction in target lesions, without a $25 \%$ enlargement in any one target lesion for a month; 3) Stable disease (SD): neither PR nor PD criteria fit; 4) Progressive disease (PD): $25 \%$ or more enlargement in target lesions or appearance of new lesions [32]. Patients were observed 24h after the CT-guided PLA for possible complications including fever, skin burns, and pain. Serum biochemical tests, including white blood count (WBC), alpha fetal protein (AFP) levels, carbohydrate antigen 199 (CA199) levels, and carcinoembryonic antigen (CEA) levels (reference range, $(4-10) * 10 \mathrm{E} 9 / \mathrm{L},(0-20) \mathrm{ng} / \mathrm{mL}$,
(0-37) $\mathrm{U} / \mathrm{mL},(0-5) \mathrm{ng} / \mathrm{mL}$, respectively), were obtained before the first PLA session and 1 week after PLA session. In addition, we also conducted a systematic review by retrieving electronic databases of PubMed, Embase and Scoups using the words as following: lung, computed tomography, metastasis, neoplasm, radiofrequency ablation, microwave ablation and laser ablation in recent five years without language limitations (see Appendix 1).

\section{RESULTS}

The basic characteristics of three cases are summarized in Table 1. These three patients were aged from 62 to 64 years. The major tumor axes ranged from $5 \mathrm{~mm}$ to $15.4 \mathrm{~mm}$. Case 1 mentioned of cough and some dyspnea after the first interventional therapy. Chest radiograph suggested small pneumothorax requiring chest tube drainage. CT scan obtained four months following the ablation showed two lesions with complete response (Figure 2A-2E and Figure 3A-3C) and one partial response (Figure 4A-4C). The WBC, AFP, CA199, and CEA levels in the patient were all normal before and after the laser ablation (Table 1). Case 2 also had a successful complete response with absent lung mass (Figure 5A-5D). Although other blood parameters were within the reference range, the level of CEA was high in both before and after the ablation. However, the patient had a good postoperative condition without discomfort in the two-month followup. Case 3 showed partial response and improved greatly after five months (Figure 6A and 6B). Most of the blood biochemical parameters were normal except for CEA level. 

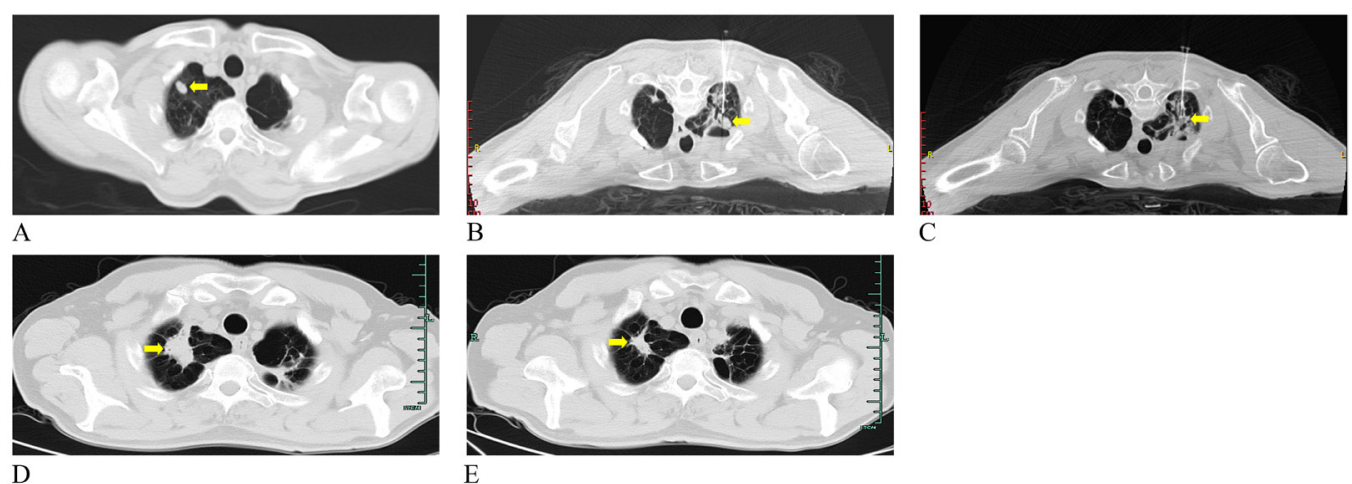

\section{$\mathrm{C}$}

Figure 2: Representative CT images from a 62-year-old man diagnosed with lung metastasis from liver cancer. A. Preoperative unenhanced CT image before PLA shows tumor about $14.5 * 7.7 \mathrm{~mm}$ in the right lung (yellow arrow). Intraoperative CT image shows the arranging needle method of using two laser fibers parallelly ablating the tumor (B. Before ablation; C. After ablation) (yellow arrows). Postoperative CT image in axial plane indicated the increased high-density lesion that has been ablated two months later $\mathbf{D}$. and reduced in four-month follow-up E. (yellow arrows).
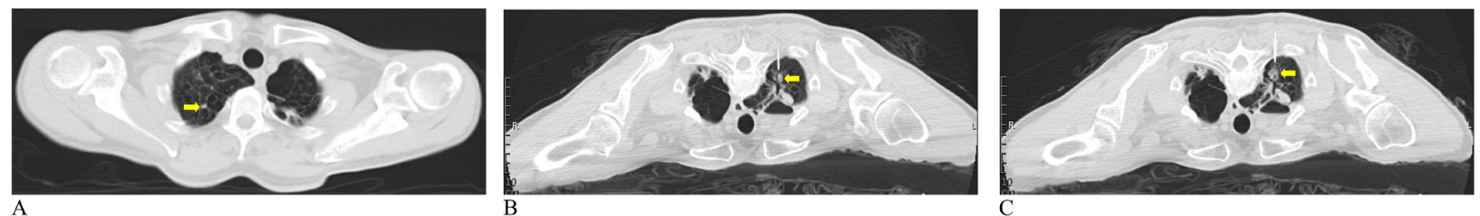

Figure 3: A. Preoperative CT image showed a mass in the upper lobe of the right lung (yellow arrow). B. A laser fiber was inserted into the location $1 \mathrm{~cm}$ away from the lesions (yellow arrow). C. CT image showed successfully ablated with high-density signal (yellow arrow).
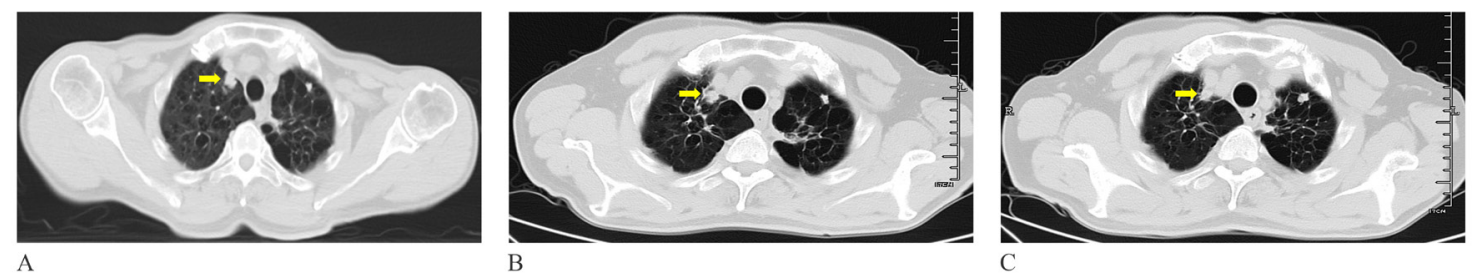

$\mathrm{C}$

Figure 4: A. Supine CT image before PLA showed the tumor measuring $15.4^{*} 10.7 \mathrm{~mm}$ in diameter adjacent to the blood vessels in the right lung (yellow arrow). B. and C. CT image two and four month obtained after ablation showed the lesion not significant change (yellow arrows).
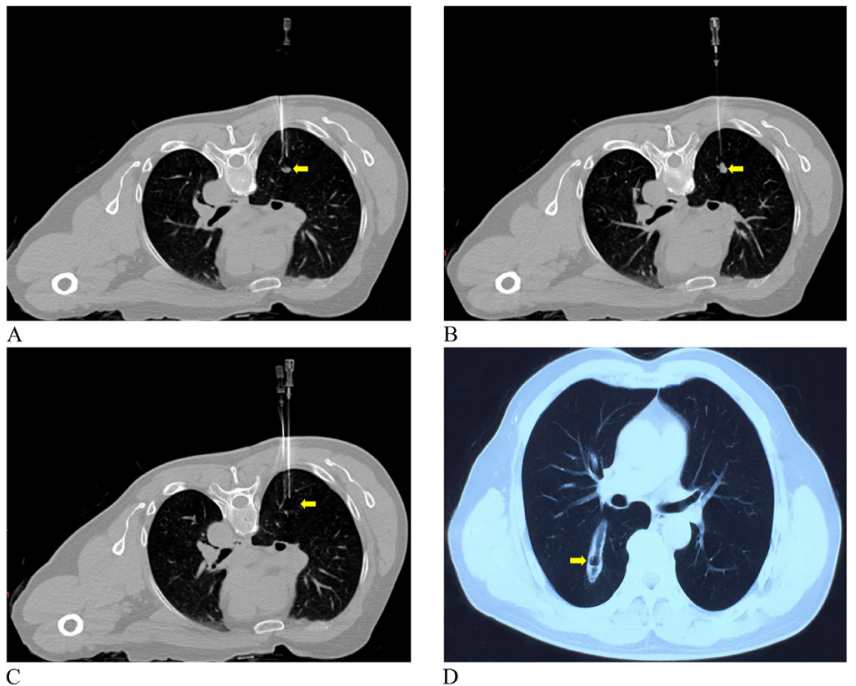

Figure 5: A 64-year-old man with lung metastasis from sigmoid colon cancer. Coronal unenhanced CT images revealed laser fibers ablating the tumor $1 \mathrm{~cm}$ outside the lesion (A. Before ablation; B. After ablation) (yellow arrows). C. CT image during the two-month follow-up demonstrated completed response (yellow arrow). 
Table 2: Summary of 962 cases with 1297 pulmonary tumors after ablation in 27 published literatures

\begin{tabular}{|c|c|c|c|c|c|c|c|c|c|c|c|}
\hline Author & Year & Country & $\begin{array}{l}\text { Characteristics of } \\
\text { patients }\end{array}$ & Treatment method & $\begin{array}{l}\text { Patients } \\
\text { (No. of } \\
\text { tumors) }\end{array}$ & \begin{tabular}{|l} 
Tumor \\
size(mm)
\end{tabular} & $\begin{array}{l}\text { Male/ } \\
\text { female }\end{array}$ & $\begin{array}{l}\text { Mean age } \\
\text { (range) }\end{array}$ & $\begin{array}{l}\text { Follow-up } \\
\text { interval } \\
\text { (months) }\end{array}$ & Prognosis & Complication \\
\hline Vogl TJ et al. & 2004 & Germany & $\begin{array}{l}24 \text { lung metastases; } \\
6 \text { localized lung } \\
\text { tumors }\end{array}$ & $\begin{array}{l}22 \text { percutaneous } \\
\text { CT-guided LA; } 8 \\
\text { percutaneous MR- } \\
\text { guided LA }\end{array}$ & $30(30)$ & $0-30$ & $14 / 16$ & $\begin{array}{l}60.2 \\
(35-81)\end{array}$ & 36 & $24 \mathrm{CR}$ & 3 pneumothorax \\
\hline $\begin{array}{l}\text { Schoellnast } \\
\mathrm{H} \text { et al. }\end{array}$ & 2011 & America & 1 NSCLC & $\begin{array}{l}\text { Percutaneous real- } \\
\text { time FDG PET CT- } \\
\text { guided RFA }\end{array}$ & $1(2)$ & $\begin{array}{l}18 * 12 \\
16^{*} 8\end{array}$ & 1 & 75 & 12 & PR & NA \\
\hline Vogl TJ et al. & 2011 & Germany & 80 lung metastases & $\begin{array}{l}\text { Percutaneous CT- } \\
\text { guided MWA }\end{array}$ & $80(130)$ & $2.9 \pm 2.4 \mathrm{~mL}$ & $30 / 50$ & \begin{tabular}{|l}
$59.7 \pm 6.4$ \\
$(48-68)$
\end{tabular} & $6-24$ & $\begin{array}{l}\text { Technical success rate: } 73.1 \% ; 1- \\
\text { and 2-year OS: } 91.3 \% \text { and } 75 \%\end{array}$ & $\begin{array}{l}11 \text { pneumothorax; } 8 \\
\text { hemorrhage; } \\
\text { hemoptysis; } \\
\text { pain;1 burn }\end{array}$ \\
\hline $\begin{array}{l}\text { Schoellnast } \\
\mathrm{H} \text { et al. }\end{array}$ & 2012 & America & $\begin{array}{l}26 \text { adenocarcinoma; } \\
9 \text { squamous cell } \\
\text { carcinoma; } 1 \text { large } \\
\text { cell carcinoma; } 3 \\
\text { NSCLC }\end{array}$ & $\begin{array}{l}\text { Percutaneous CT- } \\
\text { guided RFA }\end{array}$ & $33(39)$ & $\begin{array}{l}28 \pm 15(10- \\
75)\end{array}$ & $21 / 12$ & $75 \pm 7$ & $\begin{array}{l}19 \pm 11 \\
(1-52)\end{array}$ & $\begin{array}{l}\text { Technical success rate: } \\
97 \% ; \text { a) }<3 \mathrm{~cm} \text { median OS: } \\
24 \text { months; } 1 \text {-year OS rate: } \\
83 \% \text {; median PFS: } 11 \text { months;1- } \\
\text { year PFS rate: 50\%;median } \\
\text { TTLP: 24 months;1-year LP } \\
\text { free rate: } 65 \% \text {; b) }>3 \mathrm{~cm} \text { median } \\
\text { OS: } 15 \text { months; 1-year OS rate: } \\
56 \% ; \text { median PFS: } 5 \text { months;1-year } \\
\text { PFS rate: 11\%;median TTLP:8 } \\
\text { month; } 1 \text {-year LP free rate: } 17 \%\end{array}$ & $\begin{array}{l}1 \text { died; } 10 \\
\text { pneumothorax; } 1 \\
\text { brachial plexopathy }\end{array}$ \\
\hline $\begin{array}{ll}\text { Okuma } & \mathrm{T} \\
\text { et al. } & \end{array}$ & 2012 & Japan & $\begin{array}{lr}1 & \text { NSCLC } \\
\text { complicated } r \text { with } \\
\text { mild interstitial } \\
\text { pneumonia }\end{array}$ & $\begin{array}{l}\text { Percutaneous CT- } \\
\text { guided RFA }\end{array}$ & 1 & 23 & 1 & 67 & 11 & CR & $\begin{array}{l}1 \text { pneumothorax; } 1 \\
\text { pleural effusion }\end{array}$ \\
\hline Lu Q et al. & 2012 & China & $\begin{array}{l}48 \quad \text { NSCLC; } \\
21 \quad \text { pulmonary } \\
\text { metastasis } \\
\text { tumor }\end{array}$ & $\begin{array}{l}\text { Percutaneous CT- } \\
\text { guided MWA }\end{array}$ & $69(93)$ & $\begin{array}{l}22.3 \pm 1.7 \\
(8-55)\end{array}$ & $45 / 24$ & $65 \pm 15$ & 36 & $\begin{array}{l}\text { a)NSCLC: } 1-, 2-\text { and } 3 \text {-year } \\
\text { OS:75.0\%, } 54.2 \% \text { and } 29.2 \% ; 1-, 2- \\
\text { and 3-year recurrence-free survival } \\
\text { rates: } 72.9 \%, 50.0 \% \text { and } 27.1 \% \text {; } \\
\text { pulmonary metastatic tumor: } 1-, 2- \\
\text { and 3-year OS:47.6\%, } 23.8 \% \text { and } \\
14.3 \% ; 1-, 2-\text { and } 3 \text {-year mortality } \\
\text { rates for pulmonary metastatic } \\
\text { tumor patients: } 47.6 \%, 19.0 \% \text { and } \\
14.3 \%\end{array}$ & $\begin{array}{l}13 \text { pneumothorax;5 } \\
\text { hemoptysis; } 2 \\
\text { hemothorax;3 } \\
\text { pneumonia; } 2 \text { pain;2 } \\
\text { fever }\end{array}$ \\
\hline Liu $\mathrm{H}$ et al. & 2013 & Australia & $\begin{array}{l}5 \text { adenocarcinoma; } \\
6 \quad \text { NSCLC; } 4 \\
\text { squamous; } 1 \text { large } \\
\text { cell }\end{array}$ & $\begin{array}{l}\text { Percutaneous CT- } \\
\text { guided MWA }\end{array}$ & $15(16)$ & 24 & $11 / 4$ & $\begin{array}{l}73 \\
(52-87)\end{array}$ & $\begin{array}{l}12 \\
(6-18)\end{array}$ & 9 CR; 2 PR; 5 LTP & $\begin{array}{l}10 \text { pneumothorax; } 1 \\
\text { haemoptysis }\end{array}$ \\
\hline $\begin{array}{l}\text { Belfiore } \quad G \\
\text { et al. }\end{array}$ & 2013 & Italy & $\begin{array}{l}44 \text { lung cancer, } 25 \\
\text { lung metastases }\end{array}$ & $\begin{array}{l}\text { Percutaneous CT- } \\
\text { guided MWA }\end{array}$ & $56(69)$ & $30 \pm 9$ & $35 / 21$ & $61.5 \pm 9.13$ & 3-36 & $\begin{array}{l}\text { Mean OS: } 27.8 \pm 2.8 \text { months; } 1- \\
2-\text { and } 3 \text {-year cancer-specific } \\
\text { mortality: } 69 \%, 54 \% \text { and } 49 \%\end{array}$ & $\begin{array}{l}18 \text { pneumothorax; } \\
8 \text { pain }\end{array}$ \\
\hline Li X et al. & 2013 & China & $\begin{array}{lr}20 \text { squamous } & \text { cell } \\
\text { carcinoma; } & 26 \\
\text { adenocarcinoma; } 3 \\
\text { others }\end{array}$ & $\begin{array}{l}\text { Percutaneous CT- } \\
\text { guided RFA }\end{array}$ & $49(61)$ & $29(14-50)$ & $40 / 9$ & $\begin{array}{l}\text { Median: } 60 \\
\text { (24-82) }\end{array}$ & $\begin{array}{l}19 \\
(6-34)\end{array}$ & $\begin{array}{l}31 \text { CR; } 12 \quad \text { PR; } 6 \text { stable } \\
\text { status;median PFS: } 16 \text { weeks }\end{array}$ & $\begin{array}{l}\text { Major complication: } \\
8 \text { pneumothorax; } \\
\text { minor } \\
\text { complications: } \\
\text { self-limited minor } \\
\text { pneumothorax; } \\
\text { slight cough, fever; } \\
\text { local pain }\end{array}$ \\
\hline $\begin{array}{l}\text { Gadaleta CD } \\
\text { et al. }\end{array}$ & 2013 & Italy & $\begin{array}{lr}1 & \text { squamous } \\
\text { cell } & \text { carcinoma; } \\
2 & \text { NSCLC; } 17 \\
\text { metastatic } & \text { lung } \\
\text { cancer } & \end{array}$ & $\begin{array}{l}\text { Percutaneous CT- } \\
\text { guided RFA }\end{array}$ & $17(20)$ & $32 \pm 7$ & $6 / 11$ & $\begin{array}{l}66.5 \\
(44-81)\end{array}$ & 6 & $\begin{array}{l}11 \text { CR;technical success rate: } \\
100 \% ; \text { LTP rate: } 21 \% \text { in } 3-5-\mathrm{cm}- \\
\text { diameter tumors and } 0 \% \text { in tumors } \\
\text { of } 3 \mathrm{~cm} \text { or smaller in diameter }\end{array}$ & $\begin{array}{l}\text { Major } \\
\text { complications: } \quad 5 \\
\text { pneumothorax; } \\
1 \text { bronchopleural } \\
\text { fistula }\end{array}$ \\
\hline Yang $\mathrm{X}$ et al. & 2014 & China & $\begin{array}{l}28 \text { adenocarcinoma; } \\
13 \text { squamous; } 6 \\
\text { others }\end{array}$ & $\begin{array}{l}\text { Percutaneous CT- } \\
\text { guided MWA }\end{array}$ & 47 & $24-50$ & $30 / 17$ & $\begin{array}{l}69.4 \\
(56-82)\end{array}$ & 30 & $\begin{array}{l}\text { Median time of the first } \\
\text { recurrence,cancer-specific and } \\
\text { median OS: } 45.5,47.4 \text { and } 33.8 \\
\text { months;after MWA 1-, 2-, 3- and } \\
\text { 5-year OS rate: } 89 \%, 63 \% \text {, } 43 \% \\
\text { and } 16 \% ; \text {; after MWA 1-, 3-, 5-year } \\
\text { local control rate: } 96 \%, 64 \% \text { and } \\
48 \%\end{array}$ & $\begin{array}{l}\text { Major } \\
\text { complications: } 5 \\
\text { pneumothorax;3 } \\
\text { pleural effusion;1 } \\
\text { bronchopleural } \\
\text { fistula; minor } \\
\text { complications: 15 } \\
\text { pneumothorax;7 } \\
\text { pleural effusion;10 } \\
\text { hemoptysis; } 7 \\
\text { pneumonia }\end{array}$ \\
\hline Li XQ et al. & 2014 & China & $\begin{array}{l}36 \text { small lung } \\
\text { lesions }\end{array}$ & $\begin{array}{l}\text { Percutaneous } \\
\text { C-arm CT-guided } \\
\text { RFA }\end{array}$ & $34(36)$ & $\begin{array}{l}18 \pm 7 \\
30)\end{array}$ & $20 / 14$ & $\begin{array}{l}56 \pm 3 \\
(28-80)\end{array}$ & $\begin{array}{l}16.5 \pm 13.1 \\
(2-56)\end{array}$ & $\begin{array}{l}\text { 6-month, } 1-\text { and } 2 \text {-year OS:100.0, } \\
69.0 \text { and } 60.0 \%\end{array}$ & $\begin{array}{l}\text { 4pneumothorax; } 3 \\
\text { hemoptysis }\end{array}$ \\
\hline Hu X et al. & 2014 & China & $\begin{array}{lr}8 & \text { primary } \\
\text { lung } & \text { cancer; } 6 \\
\text { adenocarcinoma/ } & \\
\text { bronchioloalveolar; } \\
2 & \text { squamous- } \\
\text { cell rrancer; } \\
10 \quad \text { pulmonary } \\
\text { metastasis from } \\
\text { HCC r } \\
\end{array}$ & $\begin{array}{l}\text { Percutaneous CT- } \\
\text { guided RFA }\end{array}$ & $26(29)$ & $\begin{array}{l}20 \pm 4 \\
35)\end{array}$ & $19 / 7$ & $\begin{array}{l}61.3 \pm 6.7 \\
(44-77)\end{array}$ & $\begin{array}{l}13.5 \\
(3-30)\end{array}$ & $\begin{array}{l}\text { Technique efficacy rates in primary } \\
\text { tumors and lung metastases } \\
\text { patients: } 93 \% \text { and } 91.7 \% ; 2 \text {-year } \\
\text { OS rate: } 80.8 \% ; 2-\text { year-cancer- } \\
\text { specific survival rate: } 100 \% ; 1-\text { and } \\
\text { 2-year-tumor-free survival: } 69.2 \% \\
\text { and } 26.9 \%\end{array}$ & $\begin{array}{l}5 \text { pneumothorax; } 3 \\
\text { hemoptysis }\end{array}$ \\
\hline $\begin{array}{l}\text { Nour-Eldin } \\
\text { NE et al. }\end{array}$ & 2014 & Germany & $\begin{array}{l}6 \text { primary lesions; } \\
94 \text { metastatic } \\
\text { lesions }\end{array}$ & $\begin{array}{l}\text { Percutaneous CT- } \\
\text { guided RFA }\end{array}$ & $78(100)$ & NA & $46 / 32$ & 58.9 & 12 & $\begin{array}{l}80 \mathrm{CR} \text {;mean detectable time of } \\
\text { tumor residue or recurrence after } \\
\text { ablation: } 6.7 \text { months }\end{array}$ & $\begin{array}{l}8 \text { pneumothorax; } 6 \\
\text { hemorrhage; } 4 \\
\text { hemoptysis }\end{array}$ \\
\hline Iguchi $\mathrm{T}$ et al. & 2015 & Japan & 12 lung tumors & $\begin{array}{l}\text { Percutaneous CT- } \\
\text { guided RFA }\end{array}$ & $12(12)$ & $\begin{array}{l}10 \pm 3 \\
16)\end{array}$ & $6 / 6$ & $\begin{array}{l}66.4 \pm 8.6 \\
(56-81)\end{array}$ & $\begin{array}{l}19.5 \\
(3.0-41.5)\end{array}$ & $\begin{array}{l}\text { 6-month, } 1-\text { and } 2 \text {-year technique } \\
\text { efficacy rate: } 91.7 \%, 81.5 \% \text {, and } \\
81.5 \%\end{array}$ & $\begin{array}{l}3 \text { pneumothorax; } 1 \\
\text { neuralgia;1 } \\
\text { pulmonary } \\
\text { hemorrhage; } 1 \\
\text { hemothorax;1 } \\
\text { andpneumonia;1 } \\
\text { fever }\end{array}$ \\
\hline
\end{tabular}




\begin{tabular}{|c|c|c|c|c|c|c|c|c|c|c|c|}
\hline Liu L et al. & 2015 & China & lung cancer & $\begin{array}{l}\text { Percutaneous CT- } \\
\text { guided RFA }\end{array}$ & $30(30)$ & $\begin{array}{l}13.25 \pm 3.15 \\
\mathrm{~cm} 2\end{array}$ & $19 / 11$ & $\begin{array}{l}69 \\
(52-85)\end{array}$ & NA & CR or PR & NA \\
\hline $\begin{array}{l}\text { Splatt } \\
\text { et al. }\end{array}$ & 2015 & Australia & $\begin{array}{l}44 \text { NSCLC; } 26 \text { lung } \\
\text { metastasis }\end{array}$ & $\begin{array}{l}\text { Percutaneous CT- } \\
\text { guided MWA }\end{array}$ & $51(70)$ & $24.4(7-63)$ & $33 / 18$ & $\begin{array}{l}71.2 \\
(46-88)\end{array}$ & 48 & NA & $\begin{array}{l}\text { Major } \\
\text { complications:1 } \\
\text { died;9 } \\
\text { pneumothoraces;4 } \\
\text { effusion drainage;2 } \\
\text { pulmonary } \\
\text { haemorrhage; } \\
2 \text { infections; } 1 \\
\text { mechanical failure; } 1 \\
\text { chest wall burn;1 } \\
\text { pleural seeding }\end{array}$ \\
\hline Sun YH et al. & 2015 & China & $\begin{array}{lr}15 & \text { primary } \\
\text { lung cancer; } 14 \\
\text { metastatic lung } \\
\text { cancer }\end{array}$ & $\begin{array}{l}\text { Percutaneous CT- } \\
\text { guided MWA }\end{array}$ & $29(39)$ & $37(15-58)$ & $17 / 12$ & $\begin{array}{l}\text { Median: } \\
63(39-74)\end{array}$ & $\begin{array}{l}\text { Median: } 25 \\
(3-45)\end{array}$ & $\begin{array}{l}\text { 8 CR; } 14 \text { PR; } 4 \text { stable status, and } \\
3 \text { LTP;Mean PFS: } 14.6 \text { months; } 1- \\
\text { and 2-year OS: } 91.3 \% \text { and } 82.6 \%\end{array}$ & $\begin{array}{l}5 \text { pneumothorax; } 2 \\
\text { pleural effusion; } 15 \\
\text { fever }\end{array}$ \\
\hline Ni $X$ et al. & 2015 & China & $\begin{array}{l}25 \text { NSCLC; } 10 \\
\begin{array}{l}\text { squamous } \\
\text { carcinoma }\end{array}\end{array}$ & $\begin{array}{l}\text { Percutaneous CT- } \\
\text { guided MWA }\end{array}$ & $35(39)$ & $30(10-110)$ & $25 / 10$ & $\begin{array}{l}59 \\
(34-71)\end{array}$ & $\begin{array}{l}17.7 \\
(6-45)\end{array}$ & $\begin{array}{l}32 \mathrm{CR} ; 7 \text { PR;local efficacy: } 87.2 \% \text {; } \\
\text { Median MWA-related PFS, MWA- } \\
\text { related OS, PFS, and OS:5.4, 10.6, } \\
11.8 \text { and } 17.7 \text { months }\end{array}$ & $\begin{array}{l}8 \text { pneumothorax; } 6 \\
\text { pleural effusion;2 } \\
\text { pneumonia;1 } \\
\text { bronchial fistula; } 3 \\
\text { hemorrhage;9 pain }\end{array}$ \\
\hline Qi H et al. & 2015 & China & $\begin{array}{l}19 \text { right lung } \\
\text { metastases from } \\
\text { nasopharyngeal } \\
\text { carcinoma and } 10 \\
\text { left lung metastases }\end{array}$ & $\begin{array}{l}\text { Percutaneous CT- } \\
\text { guided MWA }\end{array}$ & $17(29)$ & $8-42$ & $15 / 2$ & $\begin{array}{l}45.7 \\
(28-65)\end{array}$ & $\begin{array}{l}14 \\
(3-24)\end{array}$ & 6 new metastases & $\begin{array}{l}2 \text { pneumothorax; } 4 \\
\text { parenchyma } \\
\text { bleeding }\end{array}$ \\
\hline $\begin{array}{ll}\text { Tavares } & \mathrm{E} \\
\text { Castro } & \mathrm{A} \\
\text { et al. } & \end{array}$ & 2015 & Portugal & $\begin{array}{l}20 \text { primary lung } \\
\text { cancer; } 8 \text { metastatic } \\
\text { lung cancer }\end{array}$ & $\begin{array}{l}\text { Percutaneous CT- } \\
\text { guided RFA }\end{array}$ & $28(28)$ & $\begin{array}{l}30.0 \pm 13.6 \\
(9-70)\end{array}$ & $22 / 6$ & $62 \pm 17$ & 24 & $\begin{array}{l}\text { Technical success rate: } 74.1 \% \text {;mean } \\
\text { OS: } 43 \text { months;median PFS: } 31.6 \\
\text { months;Disease-related mortality: } \\
46.4\end{array}$ & $\begin{array}{l}7 \text { pneumothorax;1 } \\
\text { pleural } \\
\text { effusion;3 mild } \\
\text { bleeding;1 } \\
\text { hemoptoic sputum;2 } \\
\text { haemorrhages }\end{array}$ \\
\hline $\begin{array}{l}\text { Acksteiner C } \\
\text { et al. }\end{array}$ & 2015 & Australia & 11 NSCLC & $\begin{array}{l}\text { Percutaneous CT- } \\
\text { guided MWA }\end{array}$ & $10(11)$ & $19(10-52)$ & $6 / 5$ & $\begin{array}{l}79 \\
(75-88)\end{array}$ & 12 & $3 \mathrm{CR} ; 5 \mathrm{PR}$ & $\begin{array}{l}2 \\
\text { pneumothorax;chest } \\
\text { tube } 3 ; 1 \text { chest wall } \\
\text { burn; } 1 \text { chest wall } \\
\text { burn;infection } 2 ; 1 \\
\text { haemoptysis;5 } \\
\text { alveolar } \\
\text { haemorrhage;2 } \\
\text { surgical emphysema }\end{array}$ \\
\hline $\begin{array}{ll}\text { Cheng } & M \\
\text { et al. } & \end{array}$ & 2016 & Australia & recurrent NSCLC & $\begin{array}{l}\text { Percutaneous } \text { CT- } \\
\text { guided RFA and } \\
\text { MWA }\end{array}$ & $12(12)$ & $34.2 \pm 12.8$ & $8 / 4$ & $71 \pm 7$ & $19 \pm 11$ & $\begin{array}{l}\text { Median OS: } 35 \text { months;mean } \\
\text { time to local progression } \\
\text { for }<30 \mathrm{~mm} \text { and }>30 \mathrm{~mm} \text { tumours: } \\
23 \text { months and } 14 \text { months }\end{array}$ & 5 pneumothorax \\
\hline $\begin{array}{l}\text { Parvizi } \quad \mathrm{N} \\
\text { et al. }\end{array}$ & 2016 & UK & $\begin{array}{lr}13 & \text { primary } \\
\text { lung cancer; } 21 \\
\text { metastatic lung } \\
\text { cancer }\end{array}$ & $\begin{array}{l}\text { Percutaneous CT- } \\
\text { guided MWA }\end{array}$ & $31(34)$ & $19(10-52)$ & $19 / 12$ & $\begin{array}{l}72.7 \\
(48-90)\end{array}$ & 12 & 7 LTP & NA \\
\hline $\begin{array}{l}\text { Fanucchi o } \\
\text { et al. }\end{array}$ & 2016 & Italy & $\begin{array}{l}25 \text { right upper } \\
\text { lobe; } 3 \text { middle } \\
\text { lobe; } 20 \text { right lower } \\
\text { lobe; } 23 \text { left upper } \\
\text { lobe;28 left lower } \\
\text { lobe }\end{array}$ & $\begin{array}{l}\text { Percutaneous CT- } \\
\text { guided RFA }\end{array}$ & $61(86)$ & 20 & $38 / 23$ & 74 & 28 & $\begin{array}{l}1-, 3-, 5 \text {-year OS rates: } 94.8 \% \\
49.0 \% \text { and } 44.5 \% ; 1-, 3-, 5 \text {-year } \\
\text { PFS rates were } 86.3 \%, 70.3 \% \text { and } \\
68.3 \%\end{array}$ & $\begin{array}{l}9 \text { pneumothorax; } 1 \\
\text { chest drainage; } 2 \\
\text { pleural effusion }\end{array}$ \\
\hline Vogl TJ et al. & 2016 & Germany & $\begin{array}{l}231 \text { colorectal lung } \\
\text { metastases }\end{array}$ & $\begin{array}{l}\text { a)Percutaneous CT- } \\
\text { guided MWA; } \\
\text { b)percutaneous CT- } \\
\text { guided LA; } \\
\text { c)percutaneous CT- } \\
\text { guided RFA }\end{array}$ & $109(231)$ & $\begin{array}{l}\text { a) } 5-50 \\
\text { b) } 10-45 \\
\text { c) } 8-42\end{array}$ & $\begin{array}{l}\text { a) } 29 / 18 \\
\text { b) } 14 / 7 \\
\text { c) } 28 / 13\end{array}$ & $\begin{array}{l}\text { a) } 64.6 \pm 11.5 \\
\text { (34-86); } \\
\text { b) } 72.9 \pm 10.4 \\
(54-94) ; \\
\text { c) } 71 \pm 10 \\
(50-90)\end{array}$ & 24 & 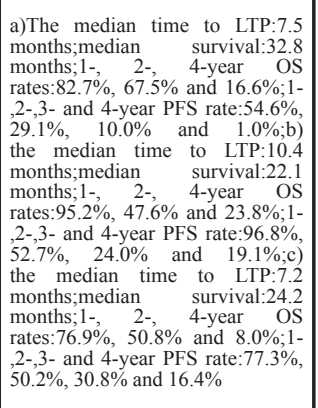 & $\begin{array}{l}\text { a) } 14 \text { pleural } \\
\text { effusion;1 } 1 \text { subcutaneous } \\
\text { emphysema;5 } \\
\text { hemorrhage;5 } \\
\text { hemoptysis;3 } \\
\text { pneumothorax;b)22 } \\
\text { pleural effusion;6 } \\
\text { subcutaneous } \\
\text { emphysema;7 } \\
\text { hemorrhage;5 } \\
\text { hemoptysis;13 } \\
\text { pneumothorax;c)37 } \\
\text { pleural effusion;31 } \\
\text { subcutaneous } \\
\text { emphysema;10 } \\
\text { hemorrhage;5 } \\
\text { hemoptysis;37 } \\
\text { pneumothorax }\end{array}$ \\
\hline $\begin{array}{l}\text { Crombé A } \\
\text { et al. }\end{array}$ & 2016 & France & 1 lung metastase & $\begin{array}{l}\text { Percutaneous CT- } \\
\text { guided RFA }\end{array}$ & $1(3)$ & $7-12$ & 1 & 82 & 120 & CR & 1 chest drainage \\
\hline
\end{tabular}

NSCLC: non-small cell lung cancer;

RFA: radiofrequency ablation;

MWA: microwave ablation;

CT: computed tomography;

PR: partial response;

CR: Complete response;

NA: not available;

OS: overall survival;

PFS: progression-free survival;

TTLP: time to local progression;

LTP: local tumor progression. 


\section{DISCUSSION}

Lung cancer is an important cause of cancer-related death in the world. Recently, emerging studies have depicted thermal ablation including RFA, MWA, PLA to be applied on the tumors. Our study mainly indicated that CT-guided PLA has significant effectiveness in the lung nidus. The therapy was optimized by using $\mathrm{CT}$ to evaluate the ablated area immediately after laser ablation. In addition, the procedure had only transient adverse effects without serious complications.

We reviewed 27 studies presenting a total of 962 cases with 1297 lung tumors following percutaneous CTguided RFA, MWA and LA. The summary of 27 studies is listed in Table $2[5-15,17-22,30,31,33-40]$. The mean age of included studies was beyond 45.7 years, and $62.2 \%$ were males. The majority of the lung tumors were completely or partially ablated. The complication rate occurred in percutaneous CT-guided RFA was $12.8 \%$ $24.6 \%[8,9]$ and in MWA was $11.9 \%-66.7 \%[5,7]$. Li et al. used CT-guided RFA percutaneously as a supplemental therapy for selected advanced non-small cell lung cancers. 31 patients had complete responses, 12 patients had partial responses, 6 patients stable disease, and no patients progressive disease [5]. However, pneumothorax occurred in 8 of 67 patients [5]. Li et al. successfully performed $\mathrm{C}$-arm CT to RFA for 36 small lung lesions. During the procedure, 4 cases had pneumothorax and 3 cases developed hemoptysis [6]. In another study, 12 patients were successfully treated by RFA via a transosseous route under CT guidance. Several complications including pneumothorax, pulmonary hemorrhage, hemothorax, pneumonia, and fever also appeared [7]. There were

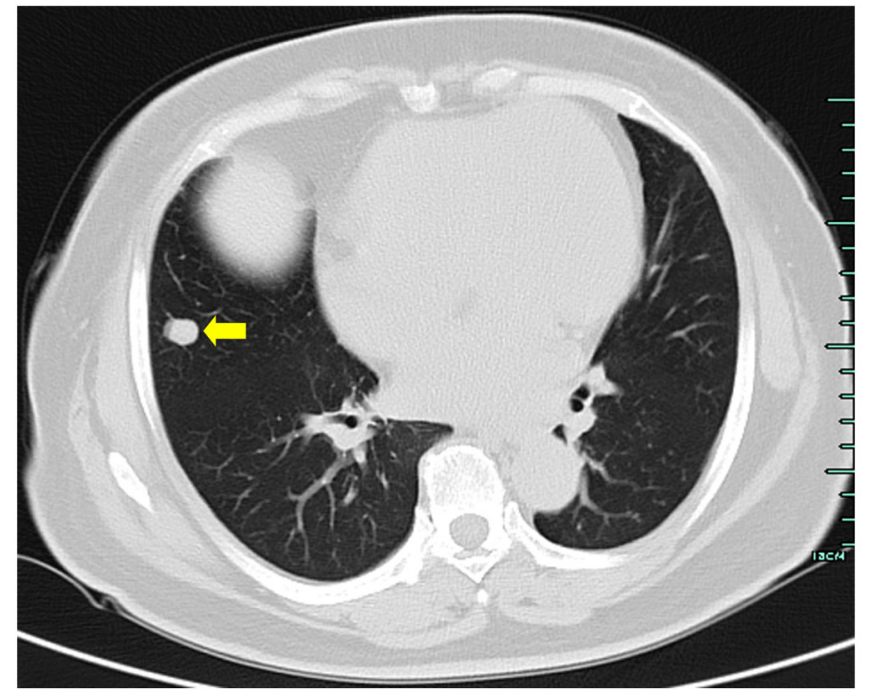

A also similar postoperative side reactions in CT-guided microwave ablation [8-10]. Previous multivariate analysis found that the prognosis in pulmonary metastatic patients treated by RFA was significantly associated with the size and the number of metastasis [41]. Compared to these thermal ablation in lung nidus, our study suggested that CT-guided PLA manifested fewer side effects and had predictable, small, and legible volumes of necrosis. These may be because PLA has thinner puncture needle, smaller thermal effect and more effective range than other thermal ablations. Laser fibers were used parallelly for larger lesions, and the combination required less energy for focal ablation and gasification. It was reported that when energy input was excessive, it might lead to malignant tumor recurrence and rapid expansion [42-44]. Therefore, in order to inactivate tumor, lower power and energy were used to produce better prognosis. Regarding the process of laser ablation in tissue, it is divided into five stages: first, tissue protein degeneration following laser-induced high temperature; second, coagulation necrosis; third, tissue liquefaction; fourth, moisture evaporation; finally, carbonizing the diseased regions. Interestingly, even if the first needle was deviating from the lesion center, we can place the second needle parallelly to ablate the whole lesions under CT guidance. Preoperative lesions showed high density imaging while it disappeared completely with low density imaging after gasification and ablation. We called this phenomenon "Lesions gasification signs". This is different from the image of laser ablation in liver lesions.

Nevertheless, there are several limitations of CT guidance:1) Imaging plane orientation for needle position is strict. 2) A small gantry bore restricts the needle length.

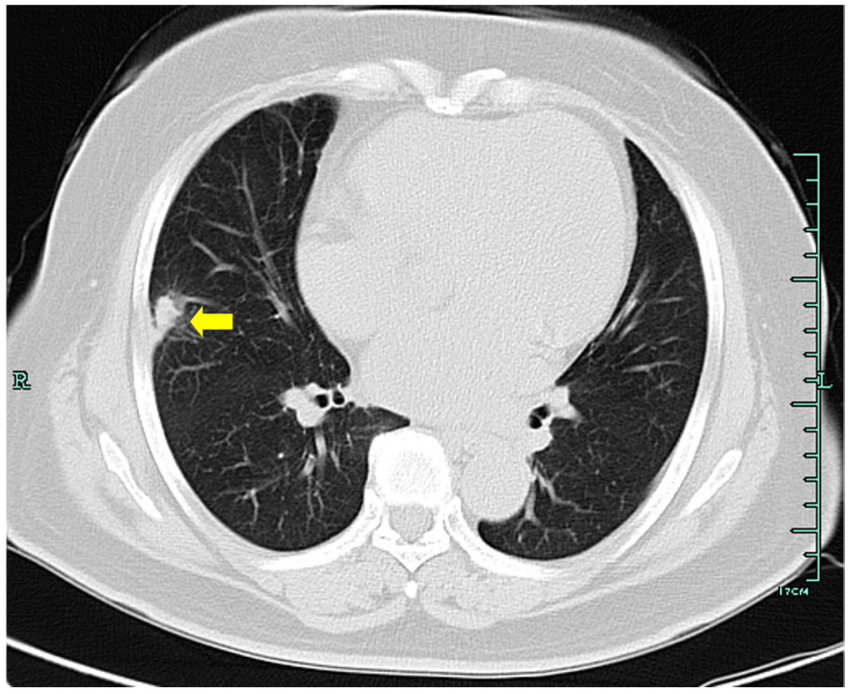

B

Figure 6: CT images from a 63-year-old woman with lung metastasis from sigmoid colon cancer. Coronal unenhanced CT images indicated the decreased tumor after PLA for lung metastasis (A. Before ablation; B. After ablation) (yellow arrows). 
3) It can not be visualised in real time [45]. In addition, the result may produce bias due to the limited sample size and period of follow-up.

In a word, this study indicated that CT-guided PLA is a minimally invasive, safe and effective method and could be promising for the treatment of lung tumors. Furthermore, especially multineedle PLA in large tumors, there are still the needs for more large-scale prospective studies to convince this in the future.

\section{ACKNOWLEDGMENTS}

This study was supported by the opening foundation of the State Key Laboratory for Diagnosis and Treatment of Infectious Diseases, Collaborative Innovation Center for Diagnosis and Treatment of Infectious Diseases, The First Affiliated Hospital of Medical College, Zhejiang University, grant NO. 2015KF06.

\section{CONFLICT OF INTERESTS}

The authors state that there is no conflict of interests regarding the publication of this paper.

\section{REFERENCES}

1. Nahleh ZA. Hormonal therapy for male breast cancer: A different approach for a different disease. Cancer treatment reviews. 2006; 32: 101-105.

2. D'Andrilli A, Maurizi G, Andreetti C, Ciccone AM, Ibrahim M, Piraino A, Mariotta S, Venuta F, Rendina EA. Sleeve Lobectomy Versus Standard Lobectomy for Lung Cancer: Functional and Oncologic Evaluation. The Annals of thoracic surgery. 2016; 101:1936-1942.

3. Pompili C, Miserocchi G. Air leak after lung resection: pathophysiology and patients' implications. Journal of thoracic disease. 2016; 8: S46-54.

4. Kim HJ, Kye BH, Lee JI, Lee SC, Lee YS, Lee IK, Kang WK, Cho HM, Moon SW, Oh ST. Surgical resection for lung metastases from colorectal cancer. Journal of the Korean Society of Coloproctology. 2010; 26: 354-358.

5. Li X, Zhao M, Wang J, Fan W, Li W, Pan T, Wu P. Percutaneous CT-guided radiofrequency ablation as supplemental therapy after systemic chemotherapy for selected advanced non-small cell lung cancers. AJR American journal of roentgenology. 2013; 201: 1362-1367.

6. Li XQ, Zhang Y, Huang DB, Zhang J, Zhang GS, Wen ZX, Li JH, Liu HL. Value of C-arm computed tomography in radiofrequency ablation of small lung lesions. Genetics and molecular research. 2014; 13: 6027-6036.

7. Iguchi T, Hiraki T, Ishii H, Gobara H, Fujiwara H, Matsui Y, Kanazawa S. Transosseous Route for CT FluoroscopyGuided Radiofrequency Ablation of Lung Tumors. Journal of vascular and interventional radiology. 2015; 26: 16941698.
8. Lu Q, Cao W, Huang L, Wan Y, Liu T, Cheng Q, Han Y, Li X. CT-guided percutaneous microwave ablation of pulmonary malignancies: Results in 69 cases. World journal of surgical oncology. 2012; 10: 80.

9. Ni X, Han JQ, Ye X, Wei ZG. Percutaneous CT-guided microwave ablation as maintenance after first-line treatment for patients with advanced NSCLC. OncoTargets and therapy. 2015; 8: 3227-3235.

10. Sun YH, Song PY, Guo Y, Sheng LJ. Computed tomography-guided percutaneous microwave ablation therapy for lung cancer. Genetics and molecular research. 2015; 14: 4858-4864.

11. Yang X, Ye X, Zheng A, Huang G, Ni X, Wang J, Han X, Li W, Wei Z. Percutaneous microwave ablation of stage I medically inoperable non-small cell lung cancer: clinical evaluation of 47 cases. Journal of surgical oncology. 2014; 110: 758-763.

12. Splatt AM, Steinke K. Major complications of high-energy microwave ablation for percutaneous CT-guided treatment of lung malignancies: Single-centre experience after 4 years. Journal of medical imaging and radiation oncology. 2015; 59: 609-616.

13. Liu H, Steinke K. High-powered percutaneous microwave ablation of stage I medically inoperable non-small cell lung cancer: a preliminary study. Journal of medical imaging and radiation oncology. 2013; 57: 466-474.

14. Belfiore G, Ronza F, Belfiore MP, Serao N, di Ronza G, Grassi R, Rotondo A. Patients' survival in lung malignancies treated by microwave ablation: our experience on 56 patients. European journal of radiology. 2013; 82: 177-181.

15. Liu L, Zhi X, Liu B, Zhang Y. Utilizing gemstone spectral CT imaging to evaluate the therapeutic efficacy of radiofrequency ablation in lung cancer. La Radiologia medica. 2015;121:261-267.

16. Cheng M, Fay M, Steinke K. Percutaneous CT-guided thermal ablation as salvage therapy for recurrent nonsmall cell lung cancer after external beam radiotherapy: A retrospective study. International journal of hyperthermia. 2016; 1-8.

17. Hu X, Zhang F, Liu XR, Wu YT, Ni YM. Efficacy and potential microRNA mechanism for computed tomographyguided percutaneous radiofrequency ablation of primary lung cancer and lung metastasis from liver cancer. Cellular physiology and biochemistry. 2014; 33: 1261-1271.

18. Nour-Eldin NE, Naguib NN, Tawfik AM, Gruber-Rouh T, Zangos S, Vogl TJ. CT volumetric assessment of pulmonary neoplasms after radiofrequency ablation: when to consider a second intervention? Journal of vascular and interventional radiology. 2014; 25: 347-354.

19. Schoellnast H, Deodhar A, Hsu M, Moskowitz C, Nehmeh SA, Thornton RH, Sofocleous CT, Alago W, Jr., Downey RJ, Azzoli CG, Rosenzweig KE, Solomon SB. Recurrent non-small cell lung cancer: evaluation of CT- 
guided radiofrequency ablation as salvage therapy. Acta radiologica. 2012; 53: 893-899.

20. Okuma T, Tsuji T, Sakamoto T, Tokura S, Akira M, Hamamoto S, Matsuoka T, Miki Y. A case of postoperative pyopneumothorax following CT-guided radiofrequency ablation for lung cancer with interstitial pneumonia. Acta radiologica short reports. 2012; 1.

21. Schoellnast H, Larson SM, Nehmeh SA, Carrasquillo JA, Thornton RH, Solomon SB. Radiofrequency ablation of non-small-cell carcinoma of the lung under real-time FDG PET CT guidance. Cardiovascular and interventional radiology. 2011; 34 Suppl 2: S182-185.

22. Gadaleta CD, Solbiati L, Mattioli V, Rubini G, Fazio V, Goffredo V, Vinciarelli G, Gadaleta-Caldarola G, Canniello E, Armenise F, D'Aluisio L, Gaudiano A, Ranieri G, et al. Unresectable lung malignancy: combination therapy with segmental pulmonary arterial chemoembolization with drug-eluting microspheres and radiofrequency ablation in 17 patients. Radiology. 2013; 267: 627-637.

23. Vogl TJ, Lehnert T, Eichler K, Proschek D, Floter J, Mack MG. Adrenal metastases: CT-guided and MR-thermometrycontrolled laser-induced interstitial thermotherapy. European radiology. 2007; 17: 2020-2027.

24. Perala J, Klemola R, Kallio R, Li C, Vihriala I, Salmela PI, Tervonen O, Sequeiros RB. MRI-guided laser ablation of neuroendocrine tumor hepatic metastases. Acta radiologica short reports. 2014; 3: 2047981613499753.

25. Wu X, Zhou P, Ma S, Tian S, Deng J, Zhao Y. [Ultrasoundguided laser thermal ablation for treatment of small liver cancer at high-risk sites]. Nan fang yi ke da xue xue bao = Journal of Southern Medical University. 2016; 36: 120-125.

26. Mauri G, Cova L, Ierace T, Baroli A, Di Mauro E, Pacella CM, Goldberg SN, Solbiati L. Treatment of Metastatic Lymph Nodes in the Neck from Papillary Thyroid Carcinoma with Percutaneous Laser Ablation. Cardiovascular and interventional radiology. 2016; 39:1023-1030.

27. Bozkurt AK, Yilmaz MF. A prospective comparison of a new cyanoacrylate glue and laser ablation for the treatment of venous insufficiency. Phlebology / Venous Forum of the Royal Society of Medicine. 2016; 31: 106-113.

28. Rao MS, Hargreaves EL, Khan AJ, Haffty BG, Danish SF. Magnetic resonance-guided laser ablation improves local control for postradiosurgery recurrence and/or radiation necrosis. Neurosurgery. 2014; 74: 658-667; discussion 667.

29. Zouari L, Bousson V, Hamze B, Roulot E, Roqueplan F, Laredo JD. CT-guided percutaneous laser photocoagulation of osteoid osteomas of the hands and feet. European radiology. 2008; 18: 2635-2641.

30. Vogl TJ, Fieguth HG, Eichler K, Straub R, Lehnert T, Zangos S, Mack M. [Laser-induced thermotherapy of lung metastases and primary lung tumors]. Der Radiologe. 2004; 44: 693-699.

31. Vogl TJ, Eckert R, Naguib NN, Beeres M, Gruber-Rouh
T, Nour-Eldin NA. Thermal Ablation of Colorectal Lung Metastases: Retrospective Comparison Among LaserInduced Thermotherapy, Radiofrequency Ablation, and Microwave Ablation. AJR American journal of roentgenology. 2016; 1-10.

32. Lockhat Z, Werke IVD, Walt BVD. Practical guidelines in the application of response evaluation criteria for solid tumours (RECIST) in oncology imaging. South African Journal of Radiology. 2004; 8.

33. Vogl TJ, Naguib NN, Gruber-Rouh T, Koitka K, Lehnert T, Nour-Eldin NE. Microwave ablation therapy: clinical utility in treatment of pulmonary metastases. Radiology. 2011; 261: 643-651.

34. Qi H, Wan C, Li X, Zhang L, Song Z, Fan W. Computed tomography-guided percutaneous microwave ablation treatment for lung metastases from nasopharyngeal carcinoma. Indian journal of cancer. 2015; 52 Suppl 2: e9195.

35. Tavares ECA, Freitas S, Portilha A, Alves F, Caseiro-Alves F. Efficacy and safety of percutaneous radiofrequency thermal ablation in the treatment of lung cancer lesions. Acta medica portuguesa. 2015; 28: 63-69.

36. Acksteiner C, Steinke K. Percutaneous microwave ablation for early-stage non-small cell lung cancer (NSCLC) in the elderly: a promising outlook. Journal of medical imaging and radiation oncology. 2015; 59: 82-90.

37. Cheng M, Fay M, Steinke K. Percutaneous CT-guided thermal ablation as salvage therapy for recurrent nonsmall cell lung cancer after external beam radiotherapy: A retrospective study. International journal of hyperthermia. 2016; 32: 316-323.

38. Parvizi N, Chung D, Little MW, Gleeson FV, Anderson EM. Does perfusion CT play a role in the evaluation of percutaneous microwave-ablated lung tumours? Clinical radiology. 2016; 71: 1137-1142.

39. Fanucchi O, Ambrogi MC, Aprile V, Cioni R, Cappelli C, Melfi F, Massimetti G, Mussi A. Long-term results of percutaneous radiofrequency ablation of pulmonary metastases: a single institution experience. Interactive cardiovascular and thoracic surgery. 2016; 23: 57-64.

40. Crombe A, Buy X, Godbert Y, Alberti N, Kind M, Bonichon F, Palussiere J. 23 Lung Metastases Treated by Radiofrequency Ablation Over 10 Years in a Single Patient: Successful Oncological Outcome of a Metastatic Cancer Without Altered Respiratory Function. Cardiovascular and interventional radiology. 2016; 39:1779-1784.

41. de Baere T. Lung tumor radiofrequency ablation: where do we stand? Cardiovascular and interventional radiology. 2011; 34: 241-251.

42. Dong S, Jian K, Kong F, Kong J, Gao J, Shan K, Wang S, Ding X, Sun W, Zheng L. Insufficient radiofrequency ablation promotes epithelial-mesenchymal transition of hepatocellular carcinoma cells through Akt and ERK signaling pathways. Journal of Translational Medicine. 
2013; 11: 1-10.

43. Brunello F, Carucci P, Gaia S, Rolle E, Brunocilla PR, Castiglione A, Ciccone G, Rizzetto M. Local Tumor Progression of Hepatocellular Carcinoma After Microwave Percutaneous Ablation: A Preliminary Report. Gastroenterology Research. 2012; 5.

44. Ohno T, Kawano K, Yokoyama H, Tahara K, Sasaki A, Aramaki M, Kitano S. Microwave coagulation therapy accelerates growth of cancer in rat liver. Journal of Hepatology. 2002; 36: 774-779.
45. Mahnken AH. CT- and MR-guided interventions in radiology. Springer. 2013. 